\title{
Validation of the Protect Questionnaire: A tool to detect mental health problems in asylum seekers by non-health professionals
}

\author{
Ricarda Mewes, PhD*, Boris Friele, Prof. Dr.**, Evert Bloemen, MD***
}

\section{Key points of interest:}

- Early recognition of mental health sequelae of torture or serious violence is of utmost importance for asylum seekers who enter the EU since such sequelae are likely to have a significant impact on the asylum procedure itself as well as rehabilitation and integration.

- It is important that non-health professionals are able to detect mental health issues using effective screening tools.

- Data presented here supports the hypothesis that the PROTECT Questionnaire is a valid and reliable tool for non-health professionals in deciding whether to offer an asylum seeker special legal procedures and whether or not to refer an asylum seeker for a clinical examination.

*) Outpatient Unit for Research, Teaching and Practice, Faculty of Psychology, University of Vienna

${ }^{\star \star}$ ) Faculty of Social Work, IUBH International University, Campus Berlin

$\left.{ }^{\star \star \star}\right)$ Pharos, Dutch Centre of Expertise on Health Disparities, Utrecht, The Netherlands

Correspondence to: b.friele@iubh-dualesstudium.de

\begin{abstract}
Introduction: Prevalence rates of trauma-related mental disorders such as posttraumatic stress disorder (PTSD) or major depression (MD) are high in asylum seekers. The PROTECT Questionnaire (PQ) was designed to detect indications of those disorders in asylum seekers. Empirical data are needed to evaluate the PQ psychometrically. The objective of this study is to investigate the reliability, validity, sensitivity, and specificity of the PQ. Method: The PQ and validated questionnaires for PTSD (Posttraumatic Diagnostic Scale, PDS) and depression (Patient Health Questionnaire-9, PHQ9) were filled in by a sample of recently arrived asylum seekers in Germany $(\mathrm{n}=141)$. A sub-sample of 91 asylum seekers took part in a structured clinical interview to diagnose PTSD or MD (SCID-I). Results: The PQ showed a one-factor structure and good reliability (Cronbach's $a=.82$ ). It correlated highly with the PDS and the PHQ-9 (rs=.53-.77; ps $\leq .001$ ). Diagnostic accuracy with regard to PTSD (AUC $=.74$; $\mathrm{SE}=.06 ; \mathrm{p}<.001 ; 95 \%-\mathrm{CI}=.63-.84)$ and $\mathrm{MD}$ $(\mathrm{AUC}=.72 ; \mathrm{SE}=.06 ; \mathrm{p}<.001 ; 95 \%-\mathrm{CI}=.61-$ .83) was adequate, suggesting an optimal cut-off of 8 or 9 . By categorizing participants into a low- and high-risk category, the PQ differentiated well between asylum seekers who fulfilled a PTSD or MD diagnosis and
\end{abstract}


those who did not. Discussion: The results support the use of the PQ as a reliable and valid instrument for the purpose of detecting signs and symptoms of the two most common mental disorders in asylum seekers. Persons found to be at risk of mental disorders should be referred to a clinical diagnostic procedure.

Keywords: asylum seekers, major depression, posttraumatic stress disorder, screening tool, validation

\section{Introduction}

The recent large influx of asylum seekers in the European Union (EU) represents a major public health challenge. Many of the asylum seekers as well as other migrants have been confronted with adverse conditions, e.g. war, violence, torture, loss of beloved persons, jobs, and property. This makes them vulnerable to negative mental health consequences. In the EU, the Asylum Procedures Directive and the Receptions Conditions Directive give guidance to member states on how to deal with the special needs of vulnerable asylum seekers (European Union, 2013). According to these directives, the needs have to be identified and taken into account with respect to reception facilities, health care provisions, and the need for special legal procedural guarantees. These requirements are based on the knowledge of high prevalence rates of mental disorders in asylum seekers and refugees. Posttraumatic Stress Disorder (PTSD) and Major Depression (MD) are among the most frequent mental disorders in asylum seekers and refugees who have experienced war, torture, or other forms of serious violence (Gäbel, Ruf, Schauer, Odenwald, \& Neuner, 2006; Ikram \& Stronks, 2016; Steel et al., 2009). The European Asylum Support
Office (EASO) considers these mental health problems as one of the indicators for special procedural needs in asylum procedures. For this reason a practical guide on evidence assessment and a digital tool were developed (EASO, 2015 \& 2016).

Evidence shows that mental health problems are associated with impairments in concentration and memory, which hinder asylum seekers from properly recounting the often traumatic background of their asylum request, resulting in wrongful asylum decisions (Cameron, 2010; Herlihy, Jobson, \& Turner, 2012; Memon, 2012; Rogers, Fox, \& Herlihy, 2014). These impairments also hinder learning capacities and can influence the person's ability to take part in social and working life in the host country. On the other hand, secure life circumstances and adequate care and treatment have considerable positive health impacts for asylum seekers (Heeren et al., 2014; Laban, Gernaat, Komproe, Schreuders, \& De Jong, 2004; Silove, Sinnerbrink, Field, Manicavasagar, \& Steel, 1997; Steel et al., 2006).

Considering the high number of steadily arriving asylum seekers, a procedure which allows for an efficient identification of vulnerable persons needing special mental health care and special procedural attention is imperative. There is a need for a tool to easily detect symptoms of mental disorders as an expression of vulnerability and special needs. Non-health professionals especially are in need of such a tool, as they are most often in contact with asylum seekers without having the clinical knowledge and experience to detect mental health problems. The PROTECT Questionnaire (PQ), investigated here, can be used by non-health staff even in less favorable situations such as asylum hotspots or detention centers. In these places, mental health is a major issue and the identification of vulnerable 
individuals is a challenge due to lack of capacity to respond and an absence of standardized tools (European Union Agency for Fundamental Rights, 2016; MSF, 2017).

Against this background, a group of specialized treatment centers for torture victims in several European countries started the PROTECT project that focused on early identification of vulnerable asylum seekers as a result of posttraumatic problems. The PROTECT project ${ }^{1}$ suggests a three-step procedure:

(1) detecting signs and symptoms of typical mental health-related sequelae of adverse experiences through a simple and short questionnaire and categorizing into risk categories;

(2) referring medium-risk and highrisk cases to an in-depth medicopsychological examination, including the diagnosis of disorders and the identification of related special needs;

(3) making known these needs in order to provide adaptations in the asylum procedure and adapted reception conditions, care, and treatment for the respective asylum seekers. With regard to step one, an easy to use screening tool is needed.

As Brewin (2005) summarizes, screening tools should be short, with items that are easy to understand, and avoid extended rating scales (e.g. use yes/no answers). They should have a clear purpose, be acceptable to respondents, and provide a simple scoring method to yield results. Focusing on short screening instruments for PTSD as one typical mental health-related sequelae in asylum seekers and refugees, Brewin (Brewin, 2005) found 14 instruments that had been subject to evaluation studies, but

http://protect-able.eu only four which consisted of fewer than 15 items. However, the BPTSD (Fullerton et al., 2000) and the SPAN scale (MeltzerBrody, Churchill, \& Davidson, 1999) require a severity scoring from 0 to 4 for each item instead of the recommended simple rating scales. The Disaster-Related Psychological Screening Test DRPST (Chou et al., 2003) was specifically designed for survivors of disasters and might not be applicable to other populations. The Primary Care PTSD Screen PC-PTSD (Prins et al., 2003) claims good predictive validity by using a very low cut-off of three or even two positive answers. Another frequently used screening instrument for PTSD in refugees (published after Brewin's review) is the Trauma Screening Questionnaire (TSQ) (e.g.(McDonald \& Sand, 2010)). However, the PC-PTSD and TSQ were developed for specific contexts. They presuppose the experience of a potentially traumatic event and were validated under this premise. A rather new questionnaire, currently attracting attention, is the Refugee Health Screener-15 (Hollifield, 2013; 2016) . The RHS-15 was designed specifically as a screening instrument for refugee populations, and good psychometric validity was found in two evaluation studies. However, despite this broad range of instruments, none of them meet the requirements for screening asylum seekers as outlined by Brewin (2005). Moreover, they do not cover signs of depression as a frequent disorder among asylum seekers (Steel et al., 2009).

\section{PROTECT Questionnaire}

The PROTECT Questionnaire (PQ) (PROTECT, 2012) was developed for adult asylum seekers to meet the demands described above. The PQ is designed to be used by non-health professionals as they 
lack the repertoire of questions to identify symptoms of mental disorders. Health professionals do not need a tool like the PQ as they have their diagnostic skills and clinical experience.

In terms of its general structure, the PQ is similar to the TSQ. Ten questions addressing specific symptoms are answered with yes or no. The questions are related to symptoms of PTSD (nightmares, anger, thinking about painful past events, being scared or frightened) and to symptoms of both MD and PTSD (sleeping problems, forgetting, losing interest, troubles concentrating). In order to account for possible cultural differences without being too specific to any particular culture, the questions of the PQ also place some emphasis on pain symptoms (headaches and non-specified pain), as it is well known that persons from non-Western cultures, as well as refugees, are more likely to experience, or at least report, somatic rather than psychological symptoms (Rohlof, Knipscheer, \& Kleber, 2014). By counting the "yes" answers a sum score is produced. The result is then assigned to one of three categories of risk for suffering from posttraumatic symptoms suggested by the PROTECT project based on clinical experience and preliminary data. A score of three or less is considered as low risk, a score of four to seven reflects a medium risk, and a score of eight or above suggests a high risk. For the medium- and high-risk categories, referral to a more in-depth examination by a psychologist or psychiatrist is recommended. Contrary to other screening instruments for PTSD, the PQ does not ask about the existence of potentially traumatic event(s). Such questions would presuppose a specific context in terms of time, trust, and expertise that is not compatible with a fast screening setting.
The present study aimed at investigating the reliability, validity, sensitivity, and specificity of the PQ in a sample of recently arrived asylum seekers in Germany.

\section{Materials and methods}

Local ethics committee approval for the study (Institutional Review Board of the Department of Psychology, University of Marburg, Germany) was obtained and all subjects provided written informed consent before participating in the study. The study was conducted in accordance with the Declaration of Helsinki. The study design was cross-sectional in nature. Data assessment took place in the German Federal state of Hesse between February 2014 and March 2015.

\section{Procedure and participants}

Asylum seekers were recruited for a study investigating the psychological health of adult asylum seekers who had been living in Germany for a maximum of one year. The study was funded by the European Refugee Fund (EFF-12-775). Asylum seekers living in Hesse, a Federal state of Germany, were approached in their accommodation or at meeting points for asylum seekers (e.g., cafés) and provided with information about the study. Asylum seekers who were willing to take part in the study were asked about several risk factors and protective factors for mental health using questionnaires and a structured clinical interview (see below).

Eligibility criteria were an age above 18 years, had been living in Germany for a maximum of 12 months, and had applied for asylum but not yet being recognized as having a right of asylum. Moreover, the participants had to be fluent in understanding and speaking (but not reading or writing) Farsi, Arabic, Kurdish, or English, or had to bring a translator of 
their language of choice. The choice of the available languages was based on data about the most frequent countries of origin and mother tongues of asylum seekers in 2014 and the years before 2014 (Bundesamt für Migration und Flüchtlinge, 2014).

\section{Assessment instruments}

All questions were provided in Farsi, Arabic, Kurdish, or English according to the participant's choice, and translators for these languages were present in case of further questions. Moreover, the software 'MultiCasi' (Knaevelsrud \& Müller, 2008) was used to present the questions and possible answer categories in a languagebased manner via a laptop with touchscreen. Questionnaires that were not available in the languages of interest were translated in a forward-backward translation process in accordance with the guidelines of Van de Vijver and Hambleton (1996).

Sociodemographic data were assessed through questions about sex, age, country of birth, mother tongue, religion, highest level of education, and the amount of time living in Germany.

Traumatic events and posttraumatic symptoms were assessed via self-report using an extended version of the Posttraumatic Diagnostic Scale (PDS) (Foa, 1995). The first part of the PDS, the trauma scale, was extended by adding items from the Harvard Trauma Questionnaire (Mollica et al., 1992) asking about traumatic events often experienced by asylum seekers. At the same time, the answer format was extended in accordance with the suggestions for traumatic events in the DSM-5 (American Psychiatric Association, 2013). Thus, it ranged from 'experienced the event myself' (highest score, 3), via 'experienced it as a witness' (2), and 'heard of it' (1), to 'none of the named possibilities' (lowest score,
$0)$. The second part of the PDS comprises questions concerning the time and impact of the most severe traumatic event. The third part consists of 17 items assessing reexperiencing, avoidance, and arousal. The total score of the third part can range from 0 to 51 , with higher scores indicating more severe posttraumatic symptomatology. The validity of the PDS has been supported in several studies. Among others, good reliability and validity for the Arabic version were found (Norris \& Aroian, 2008).

Cronbach's alpha for the PDS (third part) in the present study was .96 .

Depressive symptoms were assessed using the Patient Health Questionnaire-9 (PHQ9) (Kroenke, Spitzer, \& Williams, 2001). The PHQ-9 is a nine-item self-rating instrument, with each item representing one of the DSM-IV criteria for a depressive episode. Each item can be scored as 0 (not at all), 1 (several days), 2 (more than half the days), or 3 (nearly every day), according to the frequency of experiencing difficulties in the respective area in the previous two weeks. Sum scores range from 0 to 27 , with higher scores indicating a higher intensity of depressive symptoms (Kroenke, Spitzer, Williams, \& Lowe, 2010). The PHQ-9 is one of the most frequently used and best validated questionnaires for the assessment of depression worldwide (Löwe et al., 2004; Lowe et al., 2004). It is recommended as a general measure of depression severity by the DSM-5 (American Psychiatric Association, 2013) and has been translated into over 70 languages and dialects (Pfizer Inc., 2013). Cronbach's alpha for the PHQ-9 in the present study was .92.

In a subsample of the participants, the diagnoses Posttraumatic Stress Disorder (PTSD) and Major Depression (MD) (episode) were assessed by thoroughly trained psychologists with the help of translators. 
To this end, the respective sections of the Structured Clinical Interview for DSM-IV (SCID-I) (Wittchen, Schramm, Zaudig, \& Unland, 1997) were used. Moreover, the section for PTSD was complemented by questions assessing the newly introduced criteria for PTSD in the DSM-5 (i.e., negative cognitions and mood (American Psychiatric Association, 2013)).

\section{Statistical analyses}

SPSS 22.0 was used for all statistical analyses and only persons with full data on the respective questionnaire were included in the corresponding analyses regarding that questionnaire, i.e. persons with missing data were excluded from the relevant analyses but not from other analyses.

First, the factor structure of the PQ was analyzed using exploratory factor analysis to determine the underlying factor structure of the PQ and to have a basis for the following analyses. To test the adequacy of the items for factor analysis, the KaiserMeyer-Olkin test of sampling adequacy and Bartlett's test of sphericity were applied. A principal axis factor analysis with promax rotation was conducted that allows for intercorrelations between factors, as intercorrelations are typical for scales assessing symptoms of mental disorders. The scree plot was used to determine the number of factors to be extracted. As a measure for the reliability of the PQ total score, Cronbach's a was determined.

Second, descriptive analyses of the PQ and calculations of percentages of participants in each of the three risk categories of the PQ (low risk for a PTSD, $\mathrm{MD}$, or other trauma-associated mental disorder: sum score of 0-3; medium risk: 4-7; high risk: 8-10) were carried out.

Third, Pearson correlations between the PQ score and the measure for depression
(PHQ-9), the sum score of the extended trauma list of the PDS, and posttraumatic symptoms (PDS total symptom score) were calculated. Moreover, two t-tests for independent samples were conducted, with (a) PTSD (SCID-I criteria fulfilled vs. not) and (b) MD (SCID-I criteria fulfilled vs. not) as group variables and the sum score of the PQ as dependent variable. The variables were tested for inequality of variances using the Levene test and statistics were adapted in accordance with the results. To determine the ability of the PQ to detect persons with a PTSD or MD, the percentages of participants with or without the respective disorder in each of the three risk categories of the PQ were determined.

Fourth, to assess diagnostic accuracy, a receiver operating characteristic (ROC) curve was calculated with the PQ as predictor variable and the SCID-I diagnosis of PTSD and MD, respectively, as dependent variable.

\section{Results}

Description of the investigated sample 141 asylum seekers participated in the study. They had an average age of 32 years, and two thirds were men (see Table 1). The percentage of men precisely mirrors the percentage of all new asylum seekers in Germany in 2014 and the relatively young age is typical for asylum seekers in Germany (Bundesamt für Migration und Flüchtlinge, 2014). Most participants came from Iran, Afghanistan, Syria, or African countries and had been living in Germany for eight to nine months at the time of the study. To achieve a meaningful sample size, 16 asylum seekers who had been living in Germany for more than 12 months at the date of the assessment (up to a maximum of 42 months; their asylum cases were still ongoing) were included in the study sample. 
128 asylum seekers provided full data in the PQ (the remainder had missing data) and 91 of them took part in the structured clinical interview (SCID-I). The most frequent reasons for not taking part in the SCID were the person was no longer reachable $(n=10)$, illness $(n=8)$, no time $(\mathrm{n}=7)$, no longer interested in the study $(n=4)$, deportation $(n=2)$, or no translator available $(n=1)$. The group that did not take part in the SCID-I did not differ from the group that did take part in the SCID-I with regard to age and symptoms scores in the PQ, PDS, or PHQ-9 (all p>.16), but there were more women in the group that did not take part in the SCID-I than in the group that did take part ( $64 \%$ vs. $31 \%)$.

\section{Factor structure and reliability of the $P Q$}

The items in the present data set yielded a good Kaiser-Meyer-Olkin value of .85. The Bartlett test for sphericity was significant

Table 1: Sociodemographic characteristics $(n=141)$

\begin{tabular}{ll}
\hline $\begin{array}{l}\text { Age (years); mean } \pm \text { SD } \\
\text { Min-max }\end{array}$ & $\begin{array}{l}31.9 \pm 7.8 \\
(19-55)\end{array}$ \\
\hline Percentage men & $67 \%$ \\
\hline Highest education & \\
\hline $\begin{array}{l}\text { No graduation from formal } \\
\quad \text { schooling }\end{array}$ & $12.5 \%$ \\
$\begin{array}{l}\text { Primary school } \\
\text { Secondary school }\end{array}$ & $8.3 \%$ \\
School leaving examination or higher & $14.6 \%$ \\
Missing information & $13.4 \%$ \\
\hline Born in & \\
\hline Iran & $47.9 \%$ \\
Afghanistan & $16.0 \%$ \\
Syria & $12.5 \%$ \\
Somalia & $6.9 \%$ \\
Eritrea & $5.6 \%$ \\
Algeria & $2.1 \%$ \\
Other countries/ missing information & $9.1 \%$ \\
\hline Living in Germany for (months) & $8.5 \pm 7.5$ \\
$\quad$ mean \pm SD & \\
\hline
\end{tabular}

$\left(x^{2}(45)=335.2 ; p \leq .001\right)$, showing that the variables were appropriate for factor analysis. The scree plot favored a single-factor solution, precluding the need for a rotation strategy. The factor accounted for $39 \%$ of the variance in the items. Internal consistency can be considered as good (Cronbach's $a=.82$ ).

\section{Descriptive data on the $P Q$}

The factor analysis showed that the PQ was a one-dimensional measure. Therefore, the use of a sum score as the core descriptive value seems to be appropriate. The investigated sample had a sum score of 7.9 $(\mathrm{SD}=2.5)$ on average. Twelve participants $(9.4 \%)$ were categorized into the lowest category of vulnerability for PTSD, MD, or other trauma-associated disorders, 28 $(21.9 \%)$ were in the medium category, and $88(68.8 \%)$ were in the highest category.

\section{Relationships with other questionnaires and with diagnoses}

The PQ showed high correlations with the PHQ-9 $(r(119)=.73 ; p \leq .001)$, with the extended trauma scale of the PDS $(r(89)=.53 ; p \leq .001)$, and with the PDS total symptom score $(r(106)=.77 ; p \leq .001)$.

Participants who fulfilled the SCID-I criteria for a PTSD had significantly higher sum scores on the PQ $(8.9, \mathrm{SD}=1.5)$ than participants who did not fulfill the criteria $(6.9, \mathrm{SD}=2.7 ; t(51.6)=-4.1 ; p \leq .001)$. Likewise, participants with MD (according to the SCID-I) had higher scores $(8.9, \mathrm{SD}$ $=1.4)$ than participants without $\mathrm{MD}(6.8$, $\mathrm{SD}=2.9 ; t(42.1)=-3.99 ; p \leq .001)$.

Table 2 shows the descriptive results of the percentages of participants who fulfilled the criteria for PTSD (SCID-I) versus those who did not and their respective risk categorization according to the PQ. The PQ differentiated well between participants with PTSD versus those without in the low- and 
high-risk categories, but was only at chance level in the medium-risk category

The results for the differentiation with regard to $\mathrm{MD}$ were similar, with good differentiation in the low- and high-risk categories but at chance level in the medium category (shown in Table 3).

\section{Diagnostic accuracy}

The PQ showed adequate diagnostic accuracy with regard to PTSD (AUC=.74; $\mathrm{SE}=.06 ; \mathrm{p}<.001 ; 95 \%-\mathrm{CI}=.63-.84)$ and $\mathrm{MD}$

Table 2: Number and percentage with/without PTSD according to SCID-1

\begin{tabular}{lll}
\hline & $\begin{array}{l}\text { No Post- } \\
\text { traumatic } \\
\text { Stress } \\
\text { Disorder }\end{array}$ & $\begin{array}{l}\text { Post- } \\
\text { traumatic } \\
\text { Stress } \\
\text { Disorder }\end{array}$ \\
\hline $\begin{array}{l}\text { Low risk (0-3), } \\
\text { n (\% of category) }\end{array}$ & 7 & 1 \\
$\begin{array}{l}\text { Medium risk (4-7), } \\
\text { n (\%) }\end{array}$ & $11.5 \%)$ & $(12.5 \%)$ \\
$\begin{array}{l}\text { High risk (8-10), } \\
\text { n (\%) }\end{array}$ & $191.1 \%)$ & 7 \\
\end{tabular}

(AUC $=.72 ; \mathrm{SE}=.06 ; \mathrm{p}<.001 ; 95 \%-\mathrm{CI}=.61-$ $.83)$ in the present sample. Considering the higher importance of sensitivity than specificity for a screening instrument for the early detection of posttraumatic symptoms, cut-off scores of 8 (sensitivity was .85 for PTSD and .83 for MD; specificity was .49 for PTSD and .47 for MD) or 9 (sensitivity was .74 for PTSD and .68 for MD; specificity was .68 for PTSD and .62 for MD) might be regarded as optimal.

\section{Discussion}

The early detection of signs of mental disorders in asylum seekers is essential for
Table 3: Number and percentage with/without major depression according to SCID-1

\begin{tabular}{lll}
\hline & $\begin{array}{l}\text { No Major } \\
\text { Depression }\end{array}$ & $\begin{array}{l}\text { Major } \\
\text { Depression }\end{array}$ \\
\hline $\begin{array}{l}\text { Low risk (0-3), } \\
\text { n (\% of category) }\end{array}$ & 8 & 0 \\
Medium risk (4-7), & 8 & $(0 \%)$ \\
n (\%) & $(44.4 \%)$ & 10 \\
$\begin{array}{l}\text { High risk (8-10), } \\
\text { n (\%) }\end{array}$ & 18 & $47.6 \%)$ \\
& $(27.7 \%)$ & $(72.3 \%)$
\end{tabular}

the provision of appropriate care and for adaptations in the asylum-seeking procedure. To this end, a screening instrument, the PROTECT Questionnaire (PQ), has been developed. The current study investigated the reliability, validity, and diagnostic accuracy of the PQ.

The PQ showed a one-factor structure together with a good internal consistency. It correlated strongly with validated (but longer) questionnaires assessing symptoms of PTSD and MD. Moreover, persons with a diagnosed PTSD or MD, respectively, had significantly higher scores on the PQ than persons without the respective disorder, and the PQ showed adequate diagnostic accuracy for a short screening instrument. Using the three risk categories, the PQ differentiated well between asylum seekers who fulfilled the respective diagnosis and those who did not in the lowand high-risk categories. Additionally, the cutoff criterion for the high-risk category of a sum score of eight was confirmed by the presented analyses. However, the PQ was at chance level in the medium-risk category. Those findings are in line with the recommendation of the suggested PROTECT procedure, which is to refer all persons in the medium- and highrisk categories for a follow up and a more in-depth examination. That said, even persons in the low-risk category might have a mental 
disorder, although the probability is lower than for persons in the other categories. Those persons might benefit from re-testing.

Whereas pragmatic requirements such as 'short and simple' are crucial for screening tools, psychometric criteria cannot be ignored. In this regard, our findings are in accordance with Brewin (2005, p.59), who states that "measures with fewer items, simpler response scales, and simpler methods of scoring perform as well if not better than longer measures requiring more complex rating." Nevertheless, in order to consider specific requirements for a screening tool to be administered in the asylum procedure, it is fruitful to take into account the concerns regarding cultural sensitivity expressed by Jakobsen, Thoresen, \& Johansen (2011). They investigated the validity of the Harvard Trauma questionnaire (HTQ) and the Hopkins Symptom Checklist-25 (HSCL-25) among asylum seekers in Norway and did not find acceptable sensitivity and specificity for detecting PTSD. In accounting for these shortcomings, they emphasized the influence of differing cultural backgrounds on the performance of screening tools. This factor was also highlighted by McDonald and Sand (2010), who are in favor of an 8-step methodology for developing culturally sensitive assessment tools as suggested by Miller and colleagues (2006), and exemplified this by creating the Afghan Symptom Checklist (ASCL). However, it may not be practicable to conduct such a large scale cross-country study. At the same time, pragmatic considerations and the available evidence might complement rather than contradict each other. The PQ is a shorter and hence less demanding instrument than the HTQ and the HSCL-25 and might possibly be less influenced by cultural factors and could be much easier to translate and administer to different cultural groups. A tool that is easy to handle and distribute can be administered broadly, leading to a fairly good cost-benefit ratio. Even more importantly, it starts addressing mental problems at an early stage after arrival in the host country, thus contributing to the prevention of silencing these problems, as happens often in many of the cultures where asylum seekers come from.

As long as there is a lack of available health professionals to conduct screenings and further evaluations early in the asylum procedure, investing in mental health screening of asylum seekers by non-health professionals seems to be the best solution to improve awareness about the special needs of asylum seekers. This should eventually result in appropriate consideration of those needs in the asylum procedure and care provided. In addition, awareness and knowledge about special needs revealed by mental health screenings underlines the need to foster and support treatment facilities for asylum seekers with mental health care needs. These two challenges, i.e., a mental health screening with potential further evaluation and appropriate treatment, are closely linked to each other. However, the early documentation of symptoms of mental disorders itself, even independent of an appropriate treatment, might be of high importance for the course of the asylum application and procedure, particularly when claiming mental health concerns at any point of the administrative or judicial procedure.

\section{Limitations}

Beside the strengths of the presented study (e.g., the investigation of recently fled asylum seekers from different countries and the comparison with validated questionnaires and diagnoses), there are also some shortcomings. The current study was restricted to persons who spoke Farsi, Arabic, Kurdish, or English. Although these 
are the main languages among the current asylum seekers in Germany, generalizability to other languages and other ethnic groups is nevertheless limited. Similarly, although the lower percentage of women in the investigated sample is in line with the available data on sex percentages in asylum seekers in Germany, this may reduce the ability to transfer the findings to women.

\section{Conclusion}

The presented results support the use of the PQ as a reliable and valid instrument for the early identification of posttraumatic symptoms in asylum seekers from the main countries of origin at the time of the study. They underline the use of the PQ in the first step of the three-step procedure suggested by the PROTECT project, i.e. for the detection of signs and symptoms of typical mental health-related sequelae of severe violence and the categorization into risk categories. However, persons working with the PQ should be aware that it, like every screening instrument, only gives a first impression of symptoms and a first evaluation of the risk of suffering from depression and/or PTSD. The PQ does not replace normal diagnostic work, but contributes to finding those asylum seekers who have psychological symptoms and who do not mention their symptoms without prompting. In case of a high or medium score, those persons need to be examined more thoroughly. This clarification is even more important with regard to the legal context in which screening results could be communicated. Although the PQ seems to be successful in identifying affected persons, false-negative cases are possible. Misunderstanding the result of the PQ as a diagnosis could particularly cause harm for those individuals. Thus, the front page of the PQ contains the comment "A low risk doesn't exclude the possibility of the asylum seeker having suffered traumatic experiences". Even persons whose sum scores in the PQ are in the low risk category may benefit from a retest after a month and a short psychoeducation about possible counseling services and contact addresses. Future studies should further investigate the diagnostic accuracy of the PQ as well as its validity in other ethnic and language groups.

\section{References}

American Psychiatric Association. (2013). Diagnostic and statistical manual of mental disorders, Fifth Edition (DSM-5®).

Brewin, C. R. (2005). Systematic review of screening instruments for adults at risk of PTSD. Fournal of Traumatic Stress, 18(1), 53-62. https://doi. org/10.1002/jts. 20007

Bundesamt für Migration und Flüchtlinge (2014). Migrationsbericht des Bundesamtes für Migration und Flüchtlinge im Auftrag der Bundesregierung [Migration Report of the Federal Office for Migration and Refugees on behalf of the Federal Government] (Migrationsbericht 2014).

Cameron, H. E. (2010). Refugee Status Determinations and the Limits of Memory. International Fournal of Refugee Law, 22, 469-511. https://doi.org/10.1093/ijrl/eeq041

Chou, F. H., Su, T. T., Ou-Yang, W. C., Chien, I. C., Lu, M. K., \& Chou, P. (2003). Establishment of a disaster-related psychological screening test. Aust N Z F Psychiatry, 37(1), 97-103.

European Asylum Support Office (EASO) (2015). EASO Practical Guide: Evidence Assessment.

European Asylum Support Office (EASO) (2016). EASO tool for identification of persons with special needs.

European Union (2013). Directive 2013/32/ EU and Directive 2013/33/EU of the European Parliament and the Council of 26 June 2013. Official Fournal of the European Union, 29.6.2013 (L 180/60). https://doi. org/10.3000/19770677.L_2013.180.eng

European Union Agency for Fundamental Rights (FRA) (2016). FRA Opinion on fundamental rights in the 'hotspots' set up in Greece and Italy, 2016, 5.

Foa, E. (1995). Posttraumatic Stress Diagnostic Scale Manual. United States of America: National Computer Systems Inc. 
Fullerton, C. S., Ursano, R. J., Epstein, R. S., Crowley, B., Vance, K. L., Craig, K. J., \& Baum, A. (2000). Measurement of posttraumatic stress disorder in community samples. Nordic Fournal of Psychiatry, 54(1), 5-12. https://doi. org/10.1080/080394800427519

Gäbel, U., Ruf, M., Schauer, M., Odenwald, M., \& Neuner, F. (2006). Prävalenz der Posttraumatischen Belastungsstörung (PTSD) und Möglichkeiten der Ermittlung in der Asylverfahrenspraxis. [Prevalence of posttraumatic stress disorder among asylum seekers in Germany and its detection in the application process for asylum] Zeitschrift für Klinische Psychologie und Psychotherapie, 35(1), 1220. https://doi.org/10.1026/1616-3443.35.1.12

Heeren, M., Wittmann, L., Ehlert, U., Schnyder, U., Maier, T., \& Muller, J. (2014). Psychopathology and resident status - comparing asylum seekers, refugees, illegal migrants, labor migrants, and residents. Comprehensive Psychiatry, 55(4), 818-825. https://doi.org/10.1016/j. comppsych.2014.02.003

Herlihy, J., Jobson, L., \& Turner, S. (2012). Just tell us what happened to you: Autobiographical memory and seeking asylum. Applied Cognitive Psychology, 26(5), 661-676. https://doi. org/10.1002/acp. 2852

Hollifield, M., Toolson, E. C., Verbillis-Kolp, S., Farmer, B., Yamazaki, J., Woldehaimanot, T., \& Holland, A. (2016). Effective Screening for Emotional Distress in Refugees: The Refugee Health Screener. F Nerv Ment Dis, 204(4), 247-253. https://doi.org/10.1097/ NMD.0000000000000469

Hollifield, M., Verbillis-Kolp, S., Farmer, B., Toolson, E. C., Woldehaimanot, T., Yamazaki, J., SooHoo, J. (2013). The Refugee Health Screener-15 (RHS-15): development and validation of an instrument for anxiety, depression, and PTSD in refugees. Gen Hosp Psychiatry, 35(2), 202-209. https://doi.org/10.1016/j. genhosppsych.2012.12.002

Ikram, U., \& Stronks, K. (2016). Preserving and Improving the Mental Health of Refugees and Asylum Seekers. Den Haag: bijlage bij Briefadvies Geestelijke gezondheid van vluchtelingen [The Hague, attachment with Advisory letter mental health of refugees].

Jakobsen, M., Thoresen, S., \& Johansen, L. (2011). The Validity of Screening for Post-traumatic Stress Disorder an Other Mental Health Problems among Asylum Seekers from Different Countries. Fournal of Refugee Studies, 24(1), 171-186. https:// doi.org/10.1093/jrs/feq053
Knaevelsrud, C., \& Müller, J. (2008). MultiCASI (Multilingual Computer Assisted Self Interview). Berlin: Springer.

Kroenke, K., Spitzer, R. L., \& Williams, J. B. (2001). The PHQ-9: validity of a brief depression severity measure. f Gen Intern Med, 16(9), 606-613. https://doi.org/10.1046/j.15251497.2001.016009606.x

Kroenke, K., Spitzer, R. L., Williams, J. B., \& Lowe, B. (2010). The Patient Health Questionnaire Somatic, Anxiety, and Depressive Symptom Scales: a systematic review. Gen Hosp Psychiatry, 32(4), 345-359. https://doi.org/10.1016/j. genhosppsych.2010.03.006

Laban, C. J., Gernaat, H. B., Komproe, I. H., Schreuders, B. A., \& De Jong, J. T. (2004). Impact of a long asylum procedure on the prevalence of psychiatric disorders in Iraqi asylum seekers in The Netherlands. $\mathcal{F}$ Nerv Ment Dis, 192(12), 843-851. https://doi. org/10.1097/01.nmd.0000146739.26187.15

Löwe, B., Grafe, K., Zipfel, S., Witte, S., Loerch, B., \& Herzog, W. (2004). Diagnosing ICD10 depressive episodes: superior criterion validity of the Patient Health Questionnaire. Psychother Psychosom, 73(6), 386-390. https://doi. org/10.1159/000080384

Lowe, B., Spitzer, R. L., Grafe, K., Kroenke, K., Quenter, A., Zipfel, S., Buchholz, C., Witte, S., Herzog, W. (2004). Comparative validity of three screening questionnaires for DSM-IV depressive disorders and physicians' diagnoses. $\mathcal{F}$ Affect Disord, 78(2), 131-140. https://doi.org/10.1016/ S0165-0327(02)00237-9

McDonald, T. W., \& Sand, J. N. (2010). PostTraumatic Stress Disorder in Refugee Communities: The Importance of Culturally Sensitive Screening, Diagnosis, and Treatment. New York: Nova Science Publishers.

MSF (Medecins sans Frontieres( (2017). A Dramatic Detoriation for Asylum Seekers on Lesbos.

Meltzer-Brody, S., Churchill, E., \& Davidson, J. R. (1999). Derivation of the SPAN, a brief diagnostic screening test for post-traumatic stress disorder. Psychiatry Res, 88(1), 63-70. https://doi. org/10.1016/S0165-1781(99)00070-0

Memon, A. (2012). Credibility of Asylum Claims: Consistency and Accuracy of Autobiographical Memory Reports Following Trauma. Applied Cognitive Psychology, 26, 677-679. https://doi. org/10.1002/acp.2868

Miller, K. E., Omidian, P., Quraishy, A. S., Quraishy, N., Nasiry, M. N., Nasiry, S., Yaqubi, A.

A. (2006). The Afghan symptom checklist: a culturally grounded approach to mental 
health assessment in a conflict zone. $A m \mathcal{F}$ Orthopsychiatry, 76(4), 423-433. https:/doi. org/10.1037/0002-9432.76.4.423

Mollica, R. F., Caspi-Yavin, Y., Bollini, P., Truong, T., Tor, S., \& Lavelle, J. (1992). The Harvard Trauma Questionnaire. Validating a crosscultural instrument for measuring torture, trauma, and posttraumatic stress disorder in Indochinese refugees. $\mathcal{f}$ Nerv Ment Dis, 180(2), 111-116.

Norris, A. E., \& Aroian, K. J. (2008). Assessing reliability and validity of the Arabic language version of the Post-traumatic Diagnostic Scale (PDS) symptom items. Psychiatry Res, 160(3), 327-334. https://doi.org/10.1016/j. psychres.2007.09.005

Prins, A., Ouimette, P., Kimerling, R., Cameron, R. P., Hugelshofer, D., S., Shaw-Hegwer, J., Thrailkill, A., Gusman, F.D., Sheikh, J. I. (2003). The Primary Care PTSD Screen (PC-PTSD):

Development and operating characteristics. Primary Care Psychiatry, 9, 9-14. https://doi. org/10.1185/135525703125002360

PROTECT. (2012). Project Report. Process of Recognition and Orientation of Torture Victims in European Countries to Facilitate Care and Treatment.

Rogers, H., Fox, S., \& Herlihy, J. (2014). The importance of looking credible: the impact of the behaviourial sequelea of post-traumatic stress disorder on the credibility of asylum seekers. Psychology, Crime Eo Law, (21(2), 139-155. doi.or g/10.1080/1068316X.2014.951643

Rohlof, H. G., Knipscheer, J. W., \& Kleber, R. J. (2014). Somatization in refugees: a review. Soc Psychiatry Psychiatr Epidemiol, 49(11), 1793-1804. DOI 10.1007/s00127-014-0877-1

Silove, D., Sinnerbrink, I., Field, A., Manicavasagar, V., \& Steel, Z. (1997). Anxiety, depression and PTSD in asylum-seekers: assocations with premigration trauma and post-migration stressors. Br F Psychiatry, 170, 351-357. https://doi. org/10.1192/bjp.170.4.351

Steel, Z., Chey, T., Silove, D., Marnane, C., Bryant, R. A., \& van Ommeren, M. (2009). Association of torture and other potentially traumatic events with mental health outcomes among populations exposed to mass conflict and displacement: a systematic review and meta-analysis. $\mathcal{F} A M A$, 302(5), 537-549. https://doi.org/10.1001/ jama.2009.1132

Steel, Z., Silove, D., Brooks, R., Momartin, S., Alzuhairi, B., \& Susljik, I. (2006). Impact of immigration detention and temporary protection on the mental health of refugees. Br $\mathcal{F}$ Psychiatry,
188, 58-64. https://doi.org/10.1192/bjp. bp. 104.007864

Van de Vijver, F.J.R., \& Hambleton, R. K. (1996). Translating Tests - Some Practical Guidelines. European Psychologist, 1(2), 89-99. https://doi. org/10.1027/1016-9040.1.2.89

Wittchen, H. U., Schramm, E., Zaudig, M., \& Unland, H. (1997). SKID. Stukturiertes klinisches Interview für DSM-IV, Achse I, deutsche Version. [Structured clinical interview for DSM-IV, German Version]. Göttingen: Hogrefe. 


\section{Annex I}

\section{Questionnaire and observations for early identification of asylum seekers having suffered traumatic experiences}

The tool is not applicable to determine the legal status of a person and cannot be used to limit any claims or rights in later process.

\section{What is the purpose of the Questionnaire?}

The PROTECT Questionnaire at hand has been developped to facilitate the process of receiving asylum seekers in accordance with the directives of the European Council'.

The Questionnaire facilitates the early recognition of persons having suffered traumatic experiences, e.g. victims of torture, psychological, physical or sexual violence.

Asylum seekers having suffered such traumatic experiences should be referred to professionals of the Health Care System at an early stage in the asylum process in order to avoid deterioration and chronic manifestation of health problems and enable adaptations in reception conditions and asylum procedure.

\section{When to use the Questionnaire?}

Upon arrival in the receiving country first aid and physical shelter should be provided. It is appropriate to carry out an interview with the asylum seeker using this Questionnaire preferably after a period of rest (e.g. 7/10 days).

The Questionnaire should be applied even under difficult circumstances, rather than being neglected.

Sometimes psychological problems caused by traumatic experiences begin to appear later. That's the reason why another investigation should be carried out or the Questionnaire should be filled out a second time and the rating may have to be corrected.

1 With respect to article 17 in particular but also to articles 15 and 20 the Council Directive laying down minimum standards for the reception of asylum seekers (2003/9/EC of January $27^{\text {th }}$ 2003) and with particular respect to article $12 \S 3$ and article $13 \S 3$ indent a) of the Council Directive on minimum standards on procedures in Member States for granting and withdrawing international protection (2005/85/CE of December $\left.1^{\text {st }} 2005\right)$. 


\section{How to apply the Questionnaire?}

Before asking the set of questions, please read the following short introduction to the asylum seeker to inform him or her about the purpose of the Questionnaire and to support an environment of trust and reassurance.

The Questionnaire establishes a rating system ("low risk", "medium risk" or "high risk") for having suffered traumatic experiences.

After completing the Questionnaire a copy should be given to the asylum seeker with the recommandation that he or she submits this paper whenever meeting a Health Care System professional, a legal advisor or a reception official.

Text to be read before asking the following questions :

Dear Madam, Dear Sir,

The European Union has issued instructions to take into account the situation of some asylum seekers who need specific care.

This Questionnaire has been created jointly by specialized health and legal professionals. It will allow us to speak about your health. You can refuse to answer it.

The aim of this Questionnaire is to support you through raising awareness about your special needs.

Consequently, there are no good or bad answers to the questions and it is important that you answer as freely and naturally as possible.

Please answer the questions by YES or NO. When answering, keep in mind the experiences of the last weeks. 
Questionnaire and observations for early identification of asylum seekers having suffered traumatic experiences

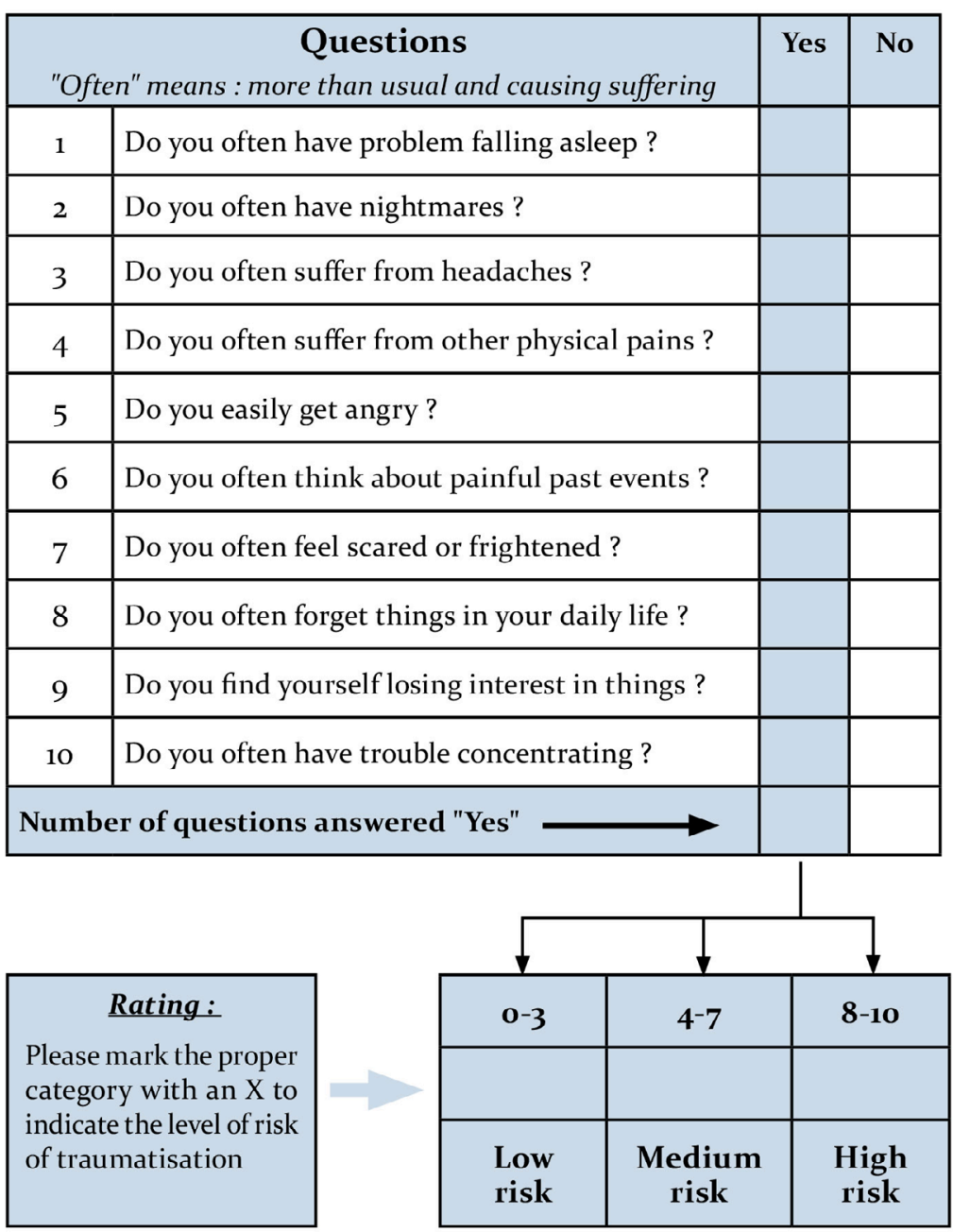


In case of a "medium risk" or a "high risk" rating the asylum seeker should be referred for medical ans psychological examination !

A "low risk" doesn't exclude the possibility of the asylum seeker having suffered traumatic experiences. Symptoms may appear later. Another screening should be carried out.

Further observations (For example : the person cries a lot, doesn't react, pays no attention... / difficulties to understand the questions / special circumstances for the interview...) :

Name of asylum seeker :

Date of birth :

Country of origin :

Date :

I agree that a copy of this document will be kept by the interviewer's organisation and can be used for statistical purpose (signature)

\section{Organisation (stamp if possible)}

After the review a copy of the Questionnaire should be given to the asylum seeker with the recommandation that he or she submits this paper whenever meeting with a Health Care System professional, a legal advisor or a reception official. 